\title{
Neural control of rising and falling tones in Mandarin speakers who stutter
}

\author{
Peter Howell $^{\mathrm{a}, *}$, Jing Jiang ${ }^{\mathrm{b}}$, Danling Peng ${ }^{\mathrm{b}}$, Chunming Lu ${ }^{\mathrm{b}, *}$ \\ a Division of Psychology and Language Sciences, University College London, UK \\ ${ }^{\mathrm{b}}$ State Key Laboratory of Cognitive Neuroscience and Learning, Beijing Normal University, Beijing, PR China
}

\section{A R T I C L E I N F O}

Article history:

Accepted 25 September 2012

Available online 2 November 2012

Keywords:

Tone control

Rising tone

Falling tone

Connectivity

Developmental stuttering

\begin{abstract}
A B S T R A C T
Neural control of rising and falling tones in Mandarin people who stutter (PWS) was examined by comparing with that which occurs in fluent speakers [Howell, Jiang, Peng, and Lu (2012). Neural control of fundamental frequency rise and fall in Mandarin tones. Brain and Language, 121(1), 35-46]. Nine PWS and nine controls were scanned. Functional connectivity analysis showed that the connections between the insula and LMC and between the LMC and the putamen differed significantly between PWS and fluent speakers during both rising and falling tones. The connection between the insula and the brainstem differed between PWS and fluent speakers only during the falling tone. These results indicated the neural control for the rising tone and the falling tone are affected in PWS. Moreover, whilst both rising and falling tones were affected in PWS, falling-tone control appeared to be affected more.
\end{abstract}

(c) 2012 Elsevier Inc. All rights reserved.

\section{Introduction}

The neural mechanisms that control rising and falling tones in fluent speakers of Mandarin have been identified recently (Howell, Jiang, Peng, \& Lu, 2012). These mechanisms may be affected in people who exhibit a range of speech disorders where laryngeal problems have been reported (Simonyan \& Horwitz, 2011). One such disorder is stuttering, and the evidence that there are laryngeal control problems in this disorder is reviewed below. Since Mandarin places high demands on laryngeal control (Xu \& Wang, 2001; Yip, 2002), an examination of whether the neural mechanisms responsible for rising-tone and falling-tone control are affected in Mandarin people who stutter (PWS) should be particularly revealing about links between laryngeal control and stuttering.

The next section of the introduction provides a brief description of the different tones in Mandarin. The evidence about speech-motor control problems in PWS is then reviewed (this mainly focuses on the laryngeal and pulmonary systems). The final section of the introduction summarizes the literature and formulates the predictions for the current study.

\subsection{The tone categories in Mandarin and the neural control of rising and falling tones}

There are four tones in Mandarin stressed syllables. These are high-flat, rising, falling-rising, and falling tones. The main differ-

\footnotetext{
* Corresponding authors. Addresses: Division of Psychology and Language Sciences, University College London, 26 Bedford Way, London WC1H 0AP, England, UK (P. Howell), State Key Laboratory of Cognitive Neuroscience and Learning, Beijing Normal University, Beijing, PR China (C. Lu). Fax: +44 (0) 2074364276 (P. Howell), fax: +86 (0) 1058806154 (C. Lu).

E-mail addresses: p.howell@ucl.ac.uk(P.Howell), luchunming@bnu.edu.cn (C. Lu).
}

ence between these tones is the voice fundamental frequency movements that arise from different laryngeal maneuvers (Harvey \& Howell, 1980; Ludlow, 2005). The high-flat and falling tones are acquired earlier than the rising and falling-rising tones (Hua \& Dodd, 2000), and more tone confusion errors occur on the rising and falling-rising tones than on the high-flat and falling tones (Shi \& Li, 2011). Neuroimaging evidence has also shown that rising-tone control involves an active mechanism, whereas falling-tone control does not (Howell et al., 2012). The neural systems that control both the rising and falling tones likely involve the left laryngeal motor cortex (LMC), insula, brainstem, and right putamen (Howell et al., 2012). The connection between the LMC and the brainstem was active during production of the rising tone, but was not active during production of the falling tone. There was an additional connection between the left insula and the LMC that showed different activity between rising and falling tones which, the authors suggested, might control whether the rise mechanism was active or not.

Mandarin speakers can change between the rising tone and the falling tone when these are required on adjacent syllables. In addition, the neural mechanisms for rising and falling tones change laryngeal control within a syllable. Consequently, the high demands on tone control in this language may reveal problems in laryngeal control in PWS that are less obvious in languages where demands on laryngeal control mechanisms are low or where laryngeal control happens over protracted periods of time (Xu \& Wang, 2001; Yip, 2002).

\subsection{Evidence for general speech motor, and laryngeal/pulmonary control deficits in PWS}

There is behavioral, physiological, and neuroimaging evidence that suggests that PWS may have problems in the speech-motor 
control of pulmonary, laryngeal, and vocal tract systems. For instance, performance during an oral kinesthesia task was poorer than in those who do not stutter (Loucks \& De Nil, 2006). The problems PWS have, in both oral kinesthesia and proprioceptive integration, interfere with sensorimotor control whilst speaking (Loucks, De Nil, \& Sasisekaran, 2007). PWS have difficulties in articulatory coordination (Howell \& Williams, 1988; Namasivayam \& Van Lieshout, 2008; Smith \& Kleinow, 2000; Smith, Sadagopan, Walsh, \& Weber-Fox, 2010). In addition, the speech-motor control problems in PWS may not be limited to articulatory systems, but may also involve systems for motor control of non-speech actions (Max, Caruso, \& Gracco, 2003) and for sequence skill learning and the development of automaticity (Smits-Bandstra \& De Nil, 2007).

Other research has shown specific problems in laryngeal control in PWS. For example, Bergmann (1986) showed that PWS had difficulty producing prosodic structure, suggesting a disturbance in laryngeal prosodic control. It has also been shown that voicing control by PWS during the production of voiceless bilabial stops that appeared to be perceptually fluent, differed from that of fluent speakers in terms of the duration of events within, as well as across, the oral and laryngeal subsystems (Max \& Gracco, 2005). Analysis of the source signal (a product of pulmonary and laryngeal control systems) showed that the disfluent voiced speech of PWS contained more aperiodic noise than did their corresponding fluent speech (Howell \& Young, 1990). This suggested a problem in laryngeal control in PWS. Other analyses of the source signal during part-word repetitions (Howell \& Vause, 1986; Howell \& Williams, 1988; Viswanath \& Neel, 1995) and direct examination of the larynx signal obtained by inverse filtering analysis (Howell \& Williams, 1992) also showed that PWS have poor laryngeal excitation during disfluent episodes. More specifically, recently it has been reported that PWS showed diminished compensation to unanticipated pitch shifts in the auditory feedback, which is consistent with difficulties in adapting pitch control to changing sensory contexts (Loucks, Chon, \& Han, 2012). To our knowledge, there are no behavioral studies that have specifically examined laryngeal control during tone production in Mandarin PWS.

General speech-motor control problems in PWS are supported by physiological evidence. For instance, an EMG study of orbicularis oris inferior muscle activity showed that PWS had higher EMG levels at speech onset and during on-going speech production than fluent speakers (Van Lieshout, Peters, Starkweather, \& Hulstijn, 1993). Activity levels in the intrinsic laryngeal muscles were lower in PWS than in fluent speakers during speech (Smith, Denny, Shaffer, Kelly, \& Hirano, 1996), and PWS showed tremor-like oscillations of EMG activity in both oral and laryngeal muscles during stuttered speech (Smith et al., 1993). These speech-motor control problems may be related to the coordination among articulatory systems, which would include the laryngeal system (Smith, 1989; Van Lieshout, Hulstijn, \& Peters, 1996).

Neuroimaging studies have examined the speech-motor control of PWS. However, they have not specifically looked at control of the laryngeal system alone or in conjunction with the pulmonary system. An early meta-analysis summarized neuroimaging studies in relation to PWS (Brown, Ingham, Ingham, Laird, \& Fox, 2005). This revealed that there were overactivations in some motor areas (primary and Supplementary motor areas motor areas), right frontal operculum/anterior insula, and right cerebellum, and underactivity in the auditory cortex in PWS (Brown et al., 2005). Other studies have reported higher or lower activity in the basal ganglia of PWS as compared to fluent speakers (Lu et al., 2010a, 2009, 2010b; Wu et al., 1995, 1997). The activity of the basal ganglia also correlated significantly with stuttering severity level (Giraud et al., 2008). Increase in gray matter volume concentration in the right basal ganglia has also been found in PWS over that observed in fluent speakers (Lu et al., 2010b). Besides regional activity differences, research has also shown altered functional or structural connections or reversed activity-sequencing between the left inferior frontal cortex (IFC) and the frontal motor areas, especially the regions for the face and larynx (Chang, Erickson, Ambrose, Hasegawa-Johnson, \& Ludlow, 2008; Chang, Horwitz, Ostuni, Reynolds, \& Ludlow, 2011; Cykowski, Fox, Ingham, Ingham, \& Robin, 2010; Lu et al., 2010a, 2009; Salmelin, Schnitzler, Schmitz, \& Freund, 2000; Sommer, Koch, Paulus, Weiller, \& Buchel, 2002; Watkins, Smith, Davis, \& Howell, 2008). Recently, studies have looked at linguistic control separately from motor control in PWS. The results confirmed that PWS had speech-motor control problems (Chang, Kenney, Loucks, \& Ludlow, 2009; Lu et al., 2010a). An additional finding was that the problems were associated with altered connectivity between the motor cortex and the cerebellum (Lu et al., 2010a, 2009).

\subsection{Summary and predictions}

Based on the behavioral, physiological, and neuroimaging evidence reviewed in Section 1.2, it was hypothesized that PWS have a general speech-motor control problem that may be evident in the laryngeal and/or pulmonary system. Because rising-tone and falling-tone control require the laryngeal and/or pulmonary system, PWS may show difference in the neural control of the rising and falling tones from fluent speakers. However, for PWS, there is little information about neural control of the larynx apart from the circumstantial evidence that suggests altered connections with the motor cortex. There is no evidence at all about control of Mandarin rising and falling tones in PWS despite the fact that such sound-contrasts may be revealing about larynx control problems in PWS in general as indicated in Section 1.1 (Xu \& Liu, 2012).

Anomalous brain activity or connectivity among brain regions in rising-tone and falling-tone control mechanisms would be expected if stuttering is associated with speech-motor control problems in the laryngeal and/or pulmonary systems. In the current study, PWS were tested on rising-tone and falling-tone control and compared against Howell et al.'s (2012) data on fluent speakers. Analyses were conducted to examine whether the connections between: (1) the left insula/right putamen and the LMC; and (2) the LMC and the brainstem, differed between fluent controls and PWS.

The predictions were as follows. Previous evidence has reported altered functional and structural connections in PWS between the left IFC or nearby regions and the frontal motor cortex, especially the regions for the face and larynx (see above). These connections are key circuits involved in control of both rising and falling tones (Howell et al., 2012; Simonyan \& Horwitz, 2011). Thus, it was hypothesized that PWS would show neural anomalies in the connections between the left insula and the LMC during both rising and falling tones. An additional prediction was based on the recent finding that the connection between the LMC and the brainstem is absent during the falling tone in fluent speakers (Howell et al., 2012). PWS have an altered connection between the motor cortex and the cerebellum during motor execution of speech (Lu et al., 2010a, 2009). Since the cerebellum and the brainstem are closely interconnected (Purves et al., 2011, p. 482), the connection between the LMC and the brainstem may also differ between fluent controls and PWS during falling-tone control.

\section{Materials and methods}

\subsection{Participants}

Nine stuttering participants (seven males) and nine fluent participants (five males) were recruited. The mean age of the PWS was 24 years (with a range of 20-29 years), and their mean time in edu- 
cation was 17 years (with a range of 15-19 years). The mean age of the fluent speakers was 24 years (with a range of 22-29 years), and their mean time in education was 15.5 (with a range of 1219 years). All participants were right-handed, native Mandarin speakers, where handedness was ascertained by the Edinburgh Handedness Inventory (Oldfield, 1971). All participants reported that they had no history of language, motor, or other neurological disorder, other than stuttering.

All PWS started stuttering during childhood, and none of them had received treatment during the year prior to this study. Stuttering severity of the PWS was assessed by a translated Mandarin version of Stuttering Severity Instrument-3 (SSI-3) (Riley, 1994). Specifically, a spontaneous speech sample of at least 300 syllables and a reading of a standard 300-syllable text (selected from a news paper) were recorded from the PWS. Any physical concomitants were noted independently by two research assistants while these recordings were made. Stuttering severity was based on these data. The severity of the PWS's stuttering ranged from very mild (lowest SSI-3 score $=13)$ to severe $($ highest SSI-3 score $=35)($ Mean $=24.44$, $S D=7.55)$. The fluent speakers participated in the study reported by Howell et al. (2012).

\subsection{Experimental task and materials}

The task and materials were the same as those used in Howell et al. (2012). In brief summary, 48 line drawings of common objects were selected from a standardized picture database (Zhang \& Yang, 2003). The Mandarin name of objects in each picture was one character long and they were distinguished by each of the four tones (high-flat, rising, falling-rising, and falling tones); there were 12 pictures for each tone. The name of the objects was controlled for age of acquisition, word frequency, and familiarity as in Howell et al. (2012). Forty-eight non-namable images were formed by randomizing the pixels of namable object-images. All pictures were randomly presented to the participants in an event-related design. The participants were scanned while they performed the task. Verbal responses were not recorded. However, the speech of the participants was monitored by the experimenter whilst scanning took place. No stuttering was observed in the PWS in this simple task.

On each trial, a picture was presented for $1 \mathrm{~s}$, and then a blank screen appeared that lasted for $2 \mathrm{~s}$. When a picture was presented on the screen, the participant had to name it aloud as fast and accurately as possible. During the baseline trials, participants were required to view the unnamable control pictures and not to make any mouth movements.

A Pentium III-based notebook with the Inquisit software package (Inquisit 2.0.4.1230, 2004, Seattle, WA: Millisecond Software) controlled stimulus presentation. An LCD projector running in $1024 \times 768$ mode displayed stimuli inside the MR control room onto a back-projection screen located at the foot of the MR scanner. Participants viewed the stimuli via a mirror attached above their eyes. Before the experiment, participants were familiarized with the procedure. The experiment commenced when participants made no errors on each tone category in the familiarization phase. The study was approved by the ethics committee of the State Key Laboratory of Cognitive Neuroscience and Learning, Beijing Normal University. Written informed consent was obtained from each participant before the experiment.

\subsection{Imaging data acquisition}

A $1.5 \mathrm{~T}$ whole-body Siemens Magnetom Sonata Maestro Class scanner (Siemens, Erlangen, Germany) was used to acquire the imaging data. During scanning, participants lay supine within the MR scanner with their heads secured by foam padding. MRI com- patible headphones were worn to reduce the background noise. Functional whole-brain, T2-weighted images were acquired using a single-shot gradient-recalled echo-planar imaging sequence $\left(\mathrm{TR}=3000 \mathrm{~ms} ; \mathrm{TE}=50 \mathrm{~ms} ;\right.$ flip angle $=90^{\circ}$; field of view, $\mathrm{FOV}=$ $220 \mathrm{~mm}$, matrix $=64 \times 64$, axial plane, resolution $=3.4 \times 3.4 \mathrm{~mm}$, slice number $=20$ slices, slice thickness $=6 \mathrm{~mm}$, slice acquisition $=$ interleave). Structural images were acquired after the functional scan using a T1-weighted MP-RAGE sequence (TR= $1970 \mathrm{~ms} ; \mathrm{TE}=3.93 \mathrm{~ms} ;$ flip angle $=15^{\circ} ; \mathrm{FOV}=220 \mathrm{~mm}$; matrix $=$ $256 \times 256$; slice number $=96$ slices; slice thickness $=1.7 \mathrm{~mm}$; saggital plane; resolution $=0.48 \times 0.48 \mathrm{~mm}$ ).

\subsection{Imaging data analysis}

\subsubsection{Preprocessing}

Preprocessing of the functional imaging data was conducted by using AFNI software (Analysis of Functional NeuroImages, http:// afni.nimh.nih.gov/afni) (Cox, 1996). Slice-time correction, image registration, motion correction, and spatial smoothing (full width at half maximum $=6 \mathrm{~mm}$ ) were performed after the first two volumes had been removed.

\subsubsection{Individual level statistics}

The preprocessed data were next fed into the generalized linear model (GLM) program to obtain the activity level for each tone category of each participant. The activity level was further scaled to percent signal change, which provided an indication of the functional activation in response to the task for each participant, as compared with the baseline (task minus baseline). Motion parameters were used at this stage to exclude potential movement artifacts. Finally, each individual image was normalized to standard Montreal Neurological Institute space.

\subsubsection{Group level differences in brain activations for the four tones in PWS}

Group level statistical tests (paired two-sample $t$-tests) were conducted on selected tone categories of PWS to see whether they were consistent with those found in fluent speakers (Howell et al., 2012) $(p<0.05$, corrected by using Monte Carlo simulation, with individual voxel $p<0.005$ and cluster size $>311 \mathrm{~mm}^{3}$ ) (Forman et al., 1995; Xiong, Gao, Lancaster, \& Fox, 1995).

\subsubsection{Group level differences in brain activations between PWS and fluent speakers in rising and falling tones}

Independent two-sample $t$-tests were conducted on rising and falling tones ( $p<0.05$, corrected) to determine whether there were neural differences between PWS and controls.

\subsubsection{Connectivity analysis using structural equation modeling (SEM)}

SEM analysis was conducted to examine the potential connection difference in the neural control of rising and falling tones between PWS and fluent speakers.

2.4.5.1. Mode setup. Four brain regions were identified that were involved in the neural control of rising and falling tones in fluent speakers, based on the previous study of Howell et al. (2012). These brain regions were also identified in the neural network responsible for laryngeal motor control (Simonyan \& Horwitz, 2011). The regions were the left $\operatorname{LMC}(x, y, z=-48,-14,40)$, insula (BA13, $x$, $y, z=-43,12,-10)$, brainstem $(x, y, z=-16,-36,-43)$, and the right putamen $(x, y, z=25,-1,-11)$. Their connections were examined in the SEM model. Based on Howell et al.'s (2012) work and other animal and imaging evidence (Simonyan \& Horwitz, 2011), it was expected that the connections between the insula and the LMC might show differences between PWS and fluent speakers in both rising and the falling tones, whereas the connection between 
the LMC and the brainstem might show differences between PWS and fluent speakers in the falling tone alone.

2.4.5.2. Preprocessing for SEM. The preprocessing was the same as that reported in Howell et al. (2012). The essential details are that the averaged time course in each brain region was calculated. The data points that corresponded to high-flat, falling-rising, and falling tones were removed, leaving those corresponding to the rising tone. The time points for high-flat, falling-rising, and falling tones were obtained by a similar procedure. Principal components analysis was conducted to identify the average pattern of responses in each brain region across all participants for each tone category (Büchel, Coull, \& Friston, 1999).

2.4.5.3. Model estimation. Parameters for the SEM model were estimated by using LISREL software 8.7 (www.ssicentral.com) (Jöreskog \& Sorbom, 1996). The estimated $\chi^{2}$, Root Mean Square Error of Approximation (RMSEA), Comparative Fit Index (CFI), and Parsimony Goodness of Fit Index (PGFI) provided an indication about how well the model fitted the data. Besides these overall fit indices, a path coefficient threshold of 0.05 , corrected for false discovery rate (FDR) was used (Genovese, Lazar, \& Nichols, 2002).

Statistical inferences about group differences in rising and falling tones were based on a stacked-models approach. The group differences for the rising tone is used to illustrate the procedure. This started with a free model, in which all path connections were allowed to vary when data from the two groups were presented. Then a restricted model was developed in which a specified connection was constrained to be equal for the two groups. To do this, first, an omnibus test was applied in which the model with all parameters constrained to be the same for the two groups (constrained model) was compared with the model without any constraints (free model). This step showed whether any of the paths between the two models was significantly different for PWS and controls, but did not specify which of the paths was actually significantly different. At this stage, the comparison of models was done by subtracting the goodness-of-fit $\chi^{2}$ value for the constrained model from the $\chi^{2}$ value for the free model. The difference $\left(\chi_{\text {diff }}^{2}\right)$ was assessed with the degrees of freedom equal to the difference in the degrees of freedom for the constrained and free models (McIntosh et al., 1994). A significant $\chi_{\text {diff }}^{2}$ indicated that at least one path differed significantly across the two groups (McIntosh \& Gonzalez-Lima, 1994a, 1994b).

When this omnibus test showed a significant difference between the two groups, the next step was to find which specific paths differed. This was done by constraining one path at a time to be the same between the two tones while other paths were unconstrained (estimated freely). Each of these models was compared with the model without any constraints by a $\chi_{\text {diff }}^{2}(d f=1)$ test. A $p$ value of 0.05 (two-tailed) was chosen as the threshold for significance (FDR corrected). The same procedure was applied to examine group differences between PWS and controls for the falling tone.

\section{Results}

\subsection{Differences between rising and falling tones in PWS}

The results showed that, as in fluent speakers (Howell et al., 2012), in PWS the rising tone differed from the falling tone. The difference was associated with the core neural structures involved in laryngeal control, i.e., the bilateral primary motor area (BA4) where the LMC is localized (Brown, Ngan, \& Liotti, 2008). The LMC showed higher activity during the rising tone than during the falling tone. This difference also covered the bilateral primary sensory area (BA3). An additional difference was that activity in the left inferior parietal cortex (BA40) was higher during the rising tone than during the falling tone (Fig. 1). No brain regions showed higher activity in the falling tone than in the rising tone. Interestingly, the left insula, putamen, and brainstem did not show differences between the rising and the falling tone in PWS, but did in fluent speakers (Howell et al., 2012). Table 1 summarizes the results.

\subsection{Differences between the rising or the falling tone and the high-flat tone in PWS}

Howell et al. (2012) made the assumption that subtracting the high-flat tone's brain activity from that of the rising or the falling tone should not affect the neural control pattern responsible for the rising tone and the falling tone respectively. This is because the high-flat tone does not involve pitch shift in either direction. This assumption was confirmed in the fluent speakers who showed stronger activity in the bilateral insula during the rising tone than during the high-flat tone, and no brain regions showed higher activity during the high-flat tone than during the rising tone (Howell et al., 2012). For the PWS, however, the same comparison indicated that the right medial frontal cortex (BA10) showed higher activity during the rising tone than during the high-flat tone (see Table 1 and the Supplemental materials, SM, Fig. S1A), whereas the insula did not. In addition, no brain regions showed higher activity during the high-flat tone than during the rising tone.

Comparison between the falling tone and the high-flat tone in PWS showed that the bilateral primary sensorimotor cortex (BA4/3), left middle frontal cortex (BA9), left inferior parietal cortex (BA40), and right declive of the cerebellum showed higher activity during the high-flat tone than during the falling tone (see Table 1 and the SM, Fig. S1B). No brain regions showed higher activity during the falling tone than during the high-flat tone. These results were different from those found in fluent speakers who showed stronger activation in the left brainstem and middle temporal cortex during the high-flat tone than the falling tone (Howell et al., 2012).

\subsection{Differences between the rising tone and the falling-rising tone and between the falling tone and the falling-rising tone in PWS}

Howell et al. (2012) reported that, for fluent speakers, neural control for the falling-rising tone was more similar to the rising tone than it was to the falling tone. Thus, subtracting the fallingrising tone from the rising tone should partially cancel activity for the rising tone whereas subtracting the falling-rising tone activity from the falling tone activity should not cancel activity for the falling tone. The comparison between the falling-rising tone and the rising tone confirmed the first prediction, as there were no differences between falling-rising and rising tones in both PWS and fluent speakers.

Stronger activations during the falling-rising tone than during the falling tone occurred for fluent speakers in the right middle frontal gyrus (BA46), right superior temporal gyrus (BA22, BA39), and left insula (BA13). Weaker activations during the falling-rising tone than during the falling tone for fluent speakers occurred for the right medial frontal gyrus (BA10) and left middle temporal gyrus (BA21). The same was reported by Howell et al. (2012). However, in PWS, higher activity during the falling-rising tone than during the falling tone was seen in the left primary motor area (BA4) and the right medial frontal cortex (BA8) (see Table 1 and the SM, Fig. S2). No brain regions showed higher activity during the falling tone than during the falling-rising tone for PWS. 


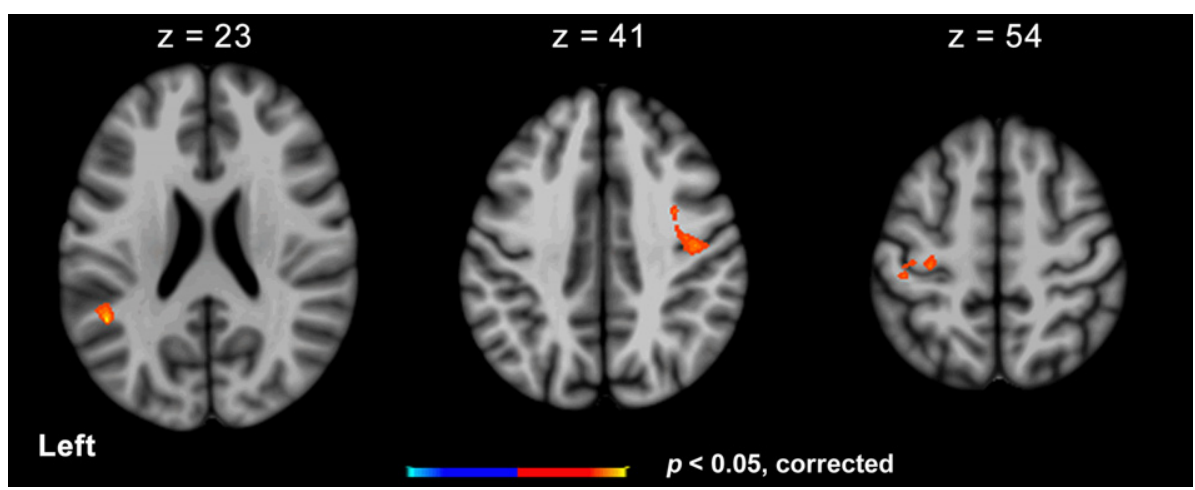

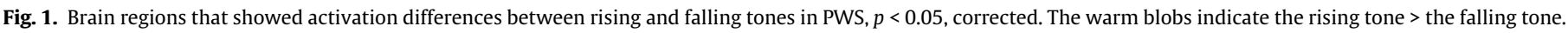

Table 1

Brain regions showing difference in brain activity between the four tones in PWS.

\begin{tabular}{|c|c|c|c|c|c|}
\hline \multirow[t]{2}{*}{ Brain region } & \multicolumn{3}{|c|}{ Position } & \multirow[t]{2}{*}{$t$-Value } & \multirow[t]{2}{*}{ Cluster volume $\left(\mathrm{mm}^{3}\right)$} \\
\hline & $x$ & $y$ & $z$ & & \\
\hline \multicolumn{6}{|l|}{ Rising $>$ falling } \\
\hline Right precentral gyrus (BA4/3) & 43 & -15 & 41 & 7.706 & 773 \\
\hline Left precentral gyrus (BA4/3) & -31 & -25 & 54 & 6.175 & 464 \\
\hline Left inferior parietal cortex (BA40) & -46 & -49 & 23 & 10.753 & 1445 \\
\hline \multicolumn{6}{|l|}{ Rising $>$ high-flat } \\
\hline Right medial frontal gyrus (BA10) & 8 & 51 & -6 & 5.821 & 333 \\
\hline \multicolumn{6}{|l|}{ Falling $<$ high-flat } \\
\hline Left middle frontal gyrus (BA9) & -54 & 25 & 31 & -12.309 & 406 \\
\hline Left pre/postcentral gyrus (BA4/3) & -61 & -6 & 27 & -7.245 & 568 \\
\hline Right pre/postcentral gyrus (BA4/3) & 43 & -17 & 45 & -5.007 & 380 \\
\hline Left suparmarginal gyrus (BA40) & -49 & -43 & 22 & -10.485 & 410 \\
\hline Right declive of the cerebellum & 12 & -65 & -18 & -8.122 & 605 \\
\hline \multicolumn{6}{|l|}{ Falling-rising $>$ falling } \\
\hline Right medial frontal gyrus (BA8) & 16 & 41 & 36 & 12.075 & 1062 \\
\hline Left precentral gyrus (BA4) & -38 & -20 & 53 & 7.594 & 1093 \\
\hline
\end{tabular}

Note: Negative results are presented in the text.

\subsection{Differences between PWS and fluent speakers for rising and falling tones}

Group comparison for the rising tone revealed that PWS showed lower activity in the bilateral premotor area (BA6) and left inferior parietal cortex (BA39) than did the fluent speakers (Fig. 2A). No brain regions showed higher activity in PWS than in fluent speakers.

Group comparison for the falling tone revealed that fluent speakers showed higher activity in the right thalamus (ventral lateral nucleus) and left cerebellum (VIII) than did PWS (see Fig. 2B). No brain regions showed higher activity in PWS than in fluent speakers (see Table 2).

\subsection{SEM results}

The SEM procedure was used to investigate the connection differences between PWS and fluent speakers in the brain regions that were involved in the neural control of tone.

3.5.1. Achievement of the best match between the model and the data 3.5.1.1. The rising tone. For the rising tone, a connection was added between the insula and the brainstem, which was not necessary in the data of fluent speakers (Howell et al., 2012). The SEM results showed that the a priori defined model was a good fit to the data of both fluent speakers $\left(\chi^{2}=1.89, d f=5, p=0.86\right)$ and PWS $\left(\chi^{2}=1.94, d f=5, p=0.86\right)$. The overall statistical fit index based on the $\chi^{2}$ value indicated that the covariance matrix was reproduced well by the model that matched best with the observed variance-covariance structure from the data (Jöreskog \& Sorbom, 1996). This result was confirmed by other overall fit statistical indices (fluent speakers, $\mathrm{RMSEA}=0.0, \quad \mathrm{PGFI}=0.49, \quad \mathrm{CFI}=1.00$; PWS, RMSEA $=0.0$, PGFI $=0.49, C F I=1.00$ ). Table 3 summarizes the standardized path coefficients for the best fitting model for each group (headed "fluent" and "stuttering") and the overall fit indices (headed with "comparison"). The reported $t$ value of these path coefficients showed that the connection between the LMC and the brainstem was significant for both groups. However, while the outward connection from the insula to the LMC was significant in fluent speakers only, the connection from the LMC to the putamen and the connection from the insula to the brainstem were significant in the PWS only. The return connection from the LMC to the insula was not significant for either group.

3.5.1.2. The falling tone. For the falling tone, a connection from the insula to the brainstem was also required for PWS. This additional connection, like its equivalent for the rising tone, was not necessary for the data of fluent speakers (Howell et al., 2012). The SEM results showed that the a priori defined model was a good fit to the data of both fluent speakers $\left(\chi^{2}=0.41, d f=5, p=0.99\right)$ and PWS $\left(\chi^{2}=1.42, d f=5, p=0.92\right)$. The model fit result was confirmed by other overall fit statistical indices (fluent speakers, RMSEA $=0.0, \quad$ PGFI $=0.50, \quad$ CFI $=1.00 ; \quad$ PWS,$\quad$ RMSEA $=0.0$, PGFI $=0.49, C F I=1.00)$. Detailed results are provided in Table 4 . 

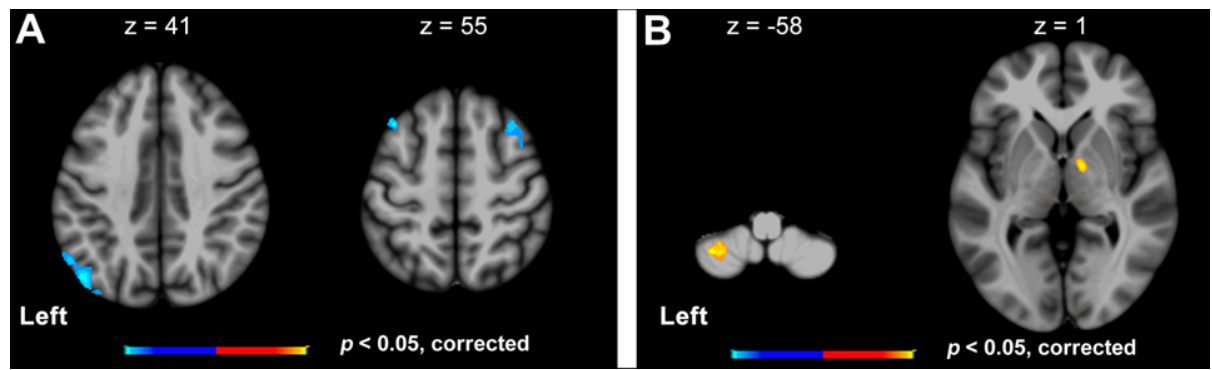

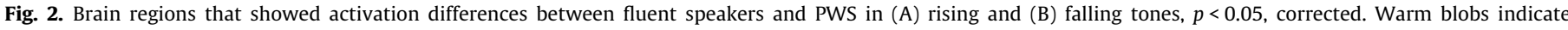
fluent $>$ stuttering, cold blobs indicate stuttering $>$ fluent.

Table 2

Brain regions showing difference in brain activity between fluent speakers and PWS.

\begin{tabular}{|c|c|c|c|c|c|}
\hline \multirow[t]{2}{*}{ Brain region } & \multicolumn{3}{|c|}{ Position } & \multirow[t]{2}{*}{$z$-Value } & \multirow[t]{2}{*}{ Cluster volume $\left(\mathrm{mm}^{3}\right)$} \\
\hline & $x$ & $y$ & $z$ & & \\
\hline \multicolumn{6}{|l|}{ Rising: fluent < stuttering } \\
\hline Right superior frontal gyrus (BA6) & 37 & 22 & 55 & -4.546 & 652 \\
\hline Left superior frontal gyrus (BA6) & -37 & 22 & 54 & -5.555 & 482 \\
\hline Left inferior parietal cortex (BA39) & -47 & -71 & 41 & -5.983 & 1813 \\
\hline \multicolumn{6}{|l|}{ Falling: fluent > stuttering } \\
\hline Right thalamus (ventral lateral nucleus) & 13 & -5 & 1 & 5.107 & 369 \\
\hline Left cerebellum (VIII) & -33 & -56 & -58 & 5.425 & 608 \\
\hline
\end{tabular}

Table 3

Standardized path coefficients for fluent speakers and PWS in the rising tone.

\begin{tabular}{|c|c|c|c|c|c|c|c|c|}
\hline \multirow[t]{2}{*}{ Paths } & \multicolumn{3}{|l|}{ Fluent } & \multicolumn{3}{|l|}{ Stuttering } & \multicolumn{2}{|c|}{ Group Comparison } \\
\hline & Standard path coefficient & $T$ & $P$ & Standard path coefficient & $T$ & $P$ & $\chi_{\text {diff }}^{2}$ & $P$ \\
\hline LMC $\rightarrow$ Insula & 0 & 0.00 & 1.000 & -0.01 & -0.02 & 0.984 & 0.00 & 1.000 \\
\hline Insula $\rightarrow$ LMC & -0.79 & -7.21 & 0.000 & 0.43 & 1.71 & 0.092 & 51.29 & 0.000 \\
\hline LMC $\rightarrow$ Putamen & -0.26 & -1.71 & 0.092 & 0.63 & 5.21 & 0.000 & 32.88 & 0.000 \\
\hline LMC $\rightarrow$ Brainstem & 0.62 & 2.69 & 0.009 & 0.38 & 4.00 & 0.000 & 1.69 & 0.194 \\
\hline Insula $\rightarrow$ Brainstem & 0.29 & 1.25 & 0.216 & 0.60 & 6.41 & 0.000 & 1.74 & 0.187 \\
\hline
\end{tabular}

Note: The bold number indicated statistically significant path coefficients and significant differences between the two groups (FDR corrected).

The reported $t$ value of these path coefficients showed that while the outward connection from the insula to the LMC was only significant in fluent speakers, the connection from the LMC to the insula and putamen and the connection from the insula to the brainstem were only significant in PWS. The connection from the LMC to the brainstem was not significant for either group.

\subsubsection{Stacked model comparison}

3.5.2.1. The rising tone. The omnibus test. The results for the rising tone showed a significant difference in path coefficients $\left(\chi_{\text {diff }}^{2}=156.92, d f=5, p<0.0001\right)$ between fluent speakers and PWS, which indicated that at least one of the paths was significantly different between the two groups.
Individual path test. Specific paths that differed between fluent speakers and PWS were located by examining the individual path coefficients (Fig. 3A). This procedure revealed that the connection from the insula to the LMC and the connection from the LMC to the putamen differed significantly between fluent speakers and PWS. Other connections did not differ significantly between the two groups.

3.5.2.2. The falling tone. The omnibus test. The results also indicated that at least one of the paths for the falling tone was significantly different between the fluent speakers and PWS $\left(\chi_{\text {diff }}^{2}=\right.$ 54.75, $d f=5, p<0.0001$ ).

Individual path test. Examination of specific paths revealed that the bi-directional connection between the insula and LMC and the

Table 4

Standardized path coefficients for fluent speakers and PWS in the falling tone.

\begin{tabular}{|c|c|c|c|c|c|c|c|c|}
\hline \multirow[t]{2}{*}{ Paths } & \multicolumn{3}{|l|}{ Fluent } & \multicolumn{3}{|l|}{ Stuttering } & \multicolumn{2}{|c|}{ Group Comparison } \\
\hline & Standard path coefficient & $T$ & $P$ & Standard path coefficient & $T$ & $P$ & $\chi_{\text {diff }}^{2}$ & $P$ \\
\hline LMC $\rightarrow$ Insula & 0 & 0.01 & 0.992 & 0.59 & 3.38 & 0.001 & 7.72 & 0.005 \\
\hline Insula $\rightarrow$ LMC & 0.7 & 5.11 & 0.000 & 0 & 0.01 & 0.992 & 14.57 & 0.000 \\
\hline LMC $\rightarrow$ Putamen & 0.04 & 0.24 & 0.811 & 0.55 & 4.19 & 0.000 & 16.62 & 0.000 \\
\hline LMC $\rightarrow$ Brainstem & -0.11 & -0.5 & 0.619 & 0.18 & 1.75 & 0.085 & 2.75 & 0.097 \\
\hline Insula $\rightarrow$ Brainstem & -0.06 & -0.3 & 0.765 & 0.73 & 7.23 & 0.000 & 8.87 & 0.003 \\
\hline
\end{tabular}

Note: The bold number indicated statistically significant path coefficients and significant differences between the two groups (FDR corrected). 
connection from the insula to the brainstem differed significantly between fluent speakers and PWS for the falling tone. The connection from the LMC to the brainstem did not differ significantly between the two groups (Fig. 3B).

\section{Discussion}

The current study obtained neural data during production of rising and falling tones in PWS according to the procedure in Howell et al. (2012). The data on PWS were then compared against Howell et al.'s data on fluent speakers. The results showed that the LMC-based neural circuits involved in laryngeal control differed between fluent speakers and PWS. The similarities and differences of the brain circuitry between PWS and fluent speakers help identify which parts act anomalously in PWS. The results are discussed further below.

\subsection{Regional activity difference between rising and falling tones}

In fluent speakers, the left insula and putamen showed stronger activity, whereas the brainstem showed weaker activity, during the rising tone than during the falling tone (Howell et al., 2012). Previous evidence has indicated that laryngeal control involves a widely distributed neural network (Simonyan \& Horwitz, 2011). In this network, the LMC may play a key role (Brown et al., 2008). The LMC is bi-directionally connected with other brain regions, including the insula, the surrounding somatosensory cortex, IFC, cingulate cortex, and the inferior parietal cortex. Additionally, the LMC is uni-directionally connected with several other brain regions including the putamen, caudate nucleus, and brainstem. Within this network, it appears that the insula, putamen, and the brainstem are closely associated with rising- and falling-tone control (Howell et al., 2012).

In PWS, however, the insula, putamen, and brainstem did not show different activity between rising and falling tones. Meanwhile, PWS showed stronger activity in the bilateral LMC and the left inferior parietal cortex during the rising tone than during the falling tone, but no brain regions showed stronger activity during the falling tone than during the rising tone. As shown in Howell et al. (2012), the LMC, insula, putamen, and brainstem constitute a circuit that is closely associated with rising- and falling-tone control. The anomalies in these brain regions during rising and falling tones suggested that the function of this neural circuit differed between fluent controls and PWS. Moreover, the results of comparison between rising and falling tones are consistent with previous evidence in that PWS generally show widely distributed overactivations in the bilateral motor cortex, including the primary motor area, supplementary motor area, and premotor area, and inferior parietal cortex (Brown et al., 2005; Lu et al., 2010a, 2009, 2010b). These findings suggest that these previously reported overactivations may be partly associated with the laryngeal control problem in PWS.

The above conclusion was supported by comparison between the rising or falling tone and the other tones (high-flat and falling-rising tones). First, the high-flat tone involves neither rising-, nor falling-tone control, and thus subtracting the high-flat tone's brain activity from that of the rising or the falling tone should not affect the neural control pattern responsible for the rising tone and the falling tone respectively. This assumption was confirmed in fluent controls. Specifically, in fluent speakers, as expected, the insula showed stronger activity during the rising tone than during the high-flat tone, whereas the brainstem showed stronger activity during the falling tone than during the high-flat tone (Howell et al., 2012). These findings further support the view that activity in these brain regions is associated with rising and falling tones. In PWS, however, only the right medial frontal gyrus survived the comparison between the rising tone and the high-flat tone, and no brain regions survived the comparison between the falling tone and the high-flat tone. In contrast, many other brain regions within the LMC-related neural circuits (the bilateral sensorimotor area, left middle frontal cortex and inferior parietal cortex, and right declive of the cerebellum) showed weaker activity during the falling tone than during the high-flat tone. The absent or reduced brain activity when the rising or falling tone were compared with the high-flat tone, further suggested that the LMC-related neural circuits that control the rising and falling tone differed between fluent controls and PWS.

Second, the comparison between the rising or falling tone and the falling-rising tone in fluent controls confirmed previous behavioral evidence that the falling-rising tone is more similar to the rising tone than to the falling tone (Hua \& Dodd, 2000), and supported the associations of brainstem/cerebellum and insula with rising and falling tones (Howell et al., 2012). In PWS, the falling-rising tone showed no activity difference with the rising tone, which is almost the same as what was found in fluent speakers. Moreover, the falling-rising tone showed stronger activity in the left LMC and right medial frontal gyrus than the falling tone. This result with PWS almost replicated the findings about the difference between the rising tone and the falling tone (see above) and confirmed the neural difference between rising-tone control and falling-tone control.
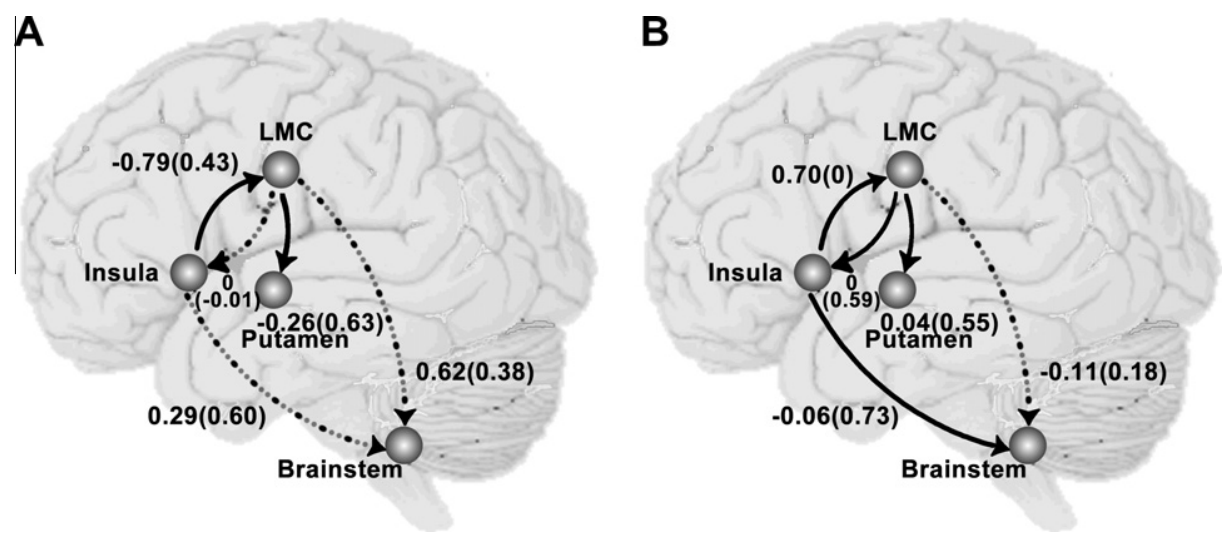

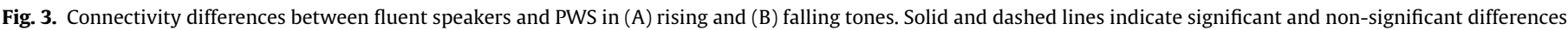

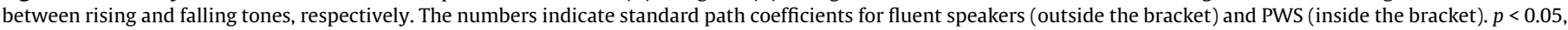
corrected. 


\subsection{Regional activity difference between PWS and fluent speakers} during rising and falling tones

It was expected that neural anomalies associated with the speech-motor control problem of PWS would be revealed by comparisons between PWS and fluent speakers. It was further expected that these neural anomalies should also occur within the LMCrelated neural network, especially those reported in Section 4.1. The results confirmed these expectations to some extent. First, during the rising tone, PWS showed higher activity than fluent speakers in the bilateral premotor cortex and the left inferior parietal cortex. As stated above, the bilateral LMC or the surrounding motor areas and the left inferior parietal cortex also showed anomalies when the rising tone and the falling tone were compared in PWS. It should be noted that the insula and putamen that were found to be associated with the rising tone did not show regional activity differences between PWS and fluent speakers. If the conclusion that these brain regions reflect the speech-motor control problem for the rising tone is reasonable, it would be surprising if they did not show up during group comparisons. However, it is possible that it is the connectivity between these brain regions, rather than their regional activity, that is more involved in the rising tone. If so, the connectivity among them would show group differences. In line with this, connectivity differences between PWS and fluent speakers were found in the SEM results, as discussed below.

Second, during the falling tone, PWS showed weaker activity in the right thalamus and the left cerebellum (VIII), and no stronger activity, than fluent speakers. These weaker neural activities may reflect the neural anomalies associated with the falling tone in PWS. Anatomicaly, the cerebellum and the brainstem are closely interconnected (Purves et al., 2011, p. 482), and through the relaying via the thalamus, they are connected with the cerebral cortex, especially the primary and premotor cortexes. Thus, the neural difference in the thalamus and cerebellum may indirectly support involvement of the brainstem during falling tones. This evidence is consistent with previous reports about abnormal activity in both the brainstem and cerebellum of PWS (Brown et al., 2005; Lu et al., 2010a; Watkins et al., 2008). Thus, the weaker activity in the thalamus and cerebellum in PWS than in fluent speakers indicated that the LMC-brainstem connection that is involved in falling-tone control in fluent speakers may be affected in PWS.

As discussed in Section 4.1, it seems that the basic neural network for laryngeal control may be inefficient in PWS. Moreover, this inefficiency seemed to be more severe in the falling tone than in the rising tone because more brain regions showed weaker activities during the falling tone than during the rising tone (see Section 4.1). Thus, it is likely that the falling tone may be affected more than the rising tone, or there is more brain plasticity that compensates for problems in rising-tone control than in falling tone control. The connections among these brain regions are discussed below.

\subsection{Neural connection comparison between PWS and fluent speakers in the rising and the falling tones}

\subsubsection{The rising tone}

As identified in Howell et al. (2012), in fluent speakers, there was a significant connection between the insula and the LMC which was negative in the rising tone but positive in the falling tone. This connection may control whether the rising-tone mechanism is active or not. This mechanism, however, was absent in PWS. That is, PWS did not show a significant connection between the left insula and the left LMC during the rising tone. In previous studies involving PWS, it has been found that the functional connections between both the frontal motor cortex and the IFC or nearby regions were altered in PWS compared to fluent speakers
(Chang et al., 2011; Lu et al., 2010a, 2010b). More importantly, diffusion tensor imaging analysis revealed reduced integrity of white matter underlying these connections (Watkins et al., 2008). Similar findings of structural anomalies have been reported in both adults (Sommer et al., 2002) and children (Chang et al., 2008; Watkins et al., 2008). These findings were taken to suggest abnormal myelogenesis in PWS that reflects a prolongation of the course of myelination in the first postnatal year (Cykowski et al., 2010). The white matter tracts in this area connect the IFC/insula and the motor cortex, as well as the posterior temporal and inferior parietal cortex, which constitute a common neural network for articulatory control, including laryngeal control, in both humans and non-humans (Fiebach, Friederici, Smith, \& Swinney, 2007; Peschke, Ziegler, Kappes, \& Baumgaertner, 2009; Simmonds, Wise, Dhanjal, \& Leech, 2011; Simonyan, Ostuni, Ludlow, \& Horwitz, 2009). The present results confirmed these findings, and further demonstrated that the anomalous functional and structural connections between the left IFC/insula and the frontal motor cortex may underlie the problem of rising-tone control in PWS.

During normal tone control, it has been hypothesized that the transfer of information input from the insula to the LMC is controlled by the insula, not the LMC. Thus, during a rising tone, the insula would become passive so as to increase the projection from the LMC to the brainstem in fluent speakers (Howell et al., 2012). If this hypothesis is reasonable, a further consequence of the altered connection between the IFC/insula and the LMC in PWS is that the motor cortex would increase its connection with other neural structures. This hypothesis was confirmed by the present results. The results showed that the connection between the LMC and the putamen was significant in PWS, but was not in fluent speakers. Thus, both the additional connection between the LMC and the putamen and the lack of connections from the insula to the LMC suggest a problem in PWS during rising-tone control possibly because of the anomalous white matter tracts that connect the IFC/ anterior insula and the motor cortex. If this conclusion is confirmed, it would suggest that these functional and structural anomalies might affect all articulatory processes rather than just the laryngeal systems.

\subsubsection{The falling tone}

As was observed with the rising tone, the connection between the insula and the LMC was absent in PWS. Such a connection has been found to be necessary to inhibit the LMC's connection with the brainstem so as to stop the laryngeal muscle activity and produce a falling tone (Howell et al., 2012). The absence of this connection suggests that the neural control mechanism to stop the laryngeal muscle activity might differ between fluent controls and PWS during the falling tone.

If the neural path from the insula, through LMC, to brainstem was affected, an alternative neural connection should appear elsewhere. As expected, PWS showed additional significant connections between the LMC and the insula and putamen, which were absent in fluent speakers. One possible explanation for these findings is that, because the connection between the left insula and the LMC was altered, the LMC lacks information for deciding how to control laryngeal muscle activity. The information that is lacking would have to be provided by other related mechanisms (i.e., brain plasticity would come into force). In PWS, the additional connection between the LMC and the insula in the falling tone compared with the fluent speakers might serve this role. Moreover, while both rising and falling tones required a connection between the insula and the brainstem when the model was fitted to the imaging data, this connection was not significant for the rising tone, but was for the falling tone, in PWS. This connection did not exist in the data for fluent speakers either (Howell et al., 2012). Since there is no direct behavioral evidence to support the explanation 
A

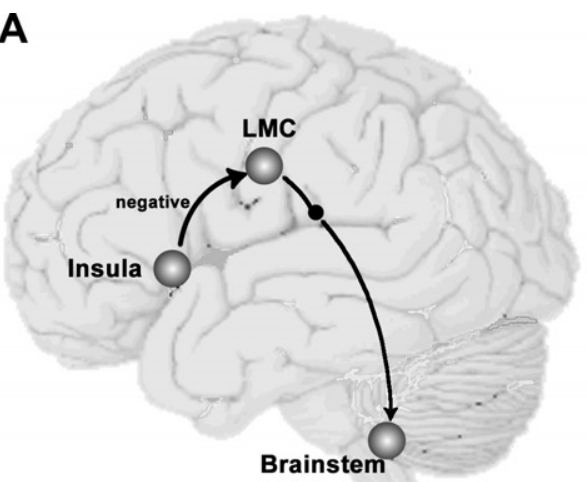

C

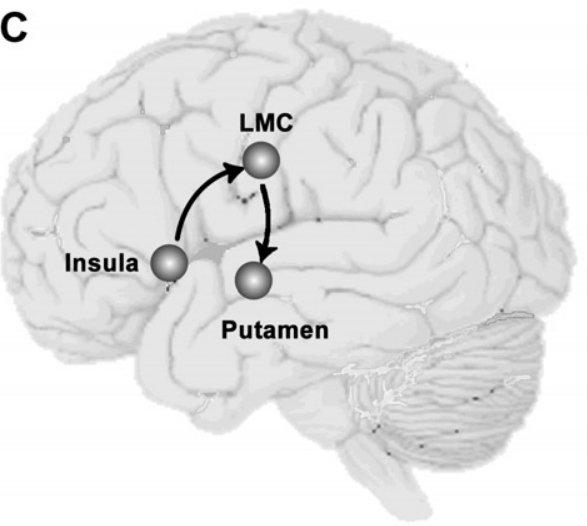

E

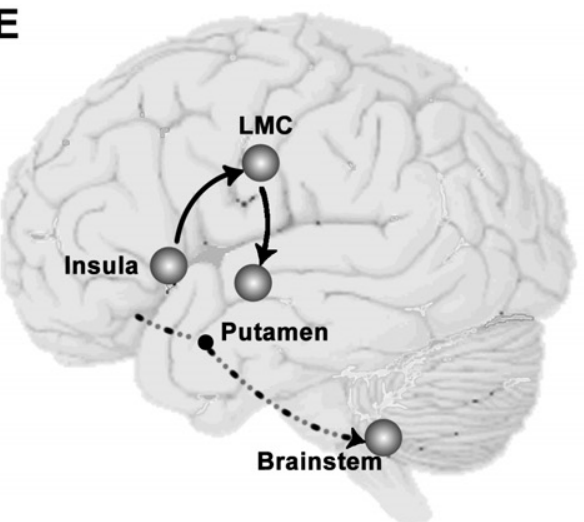

B

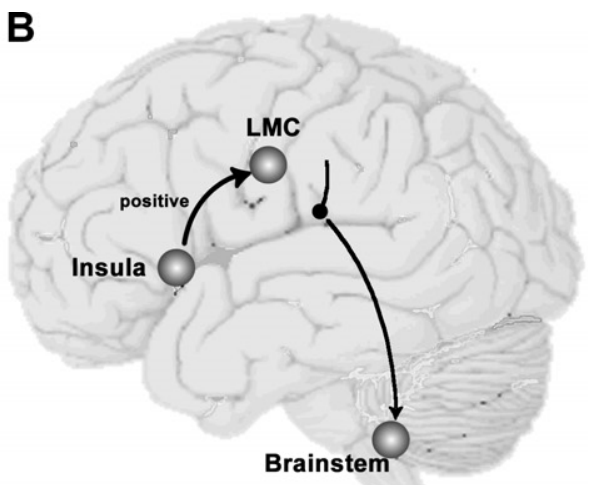

D

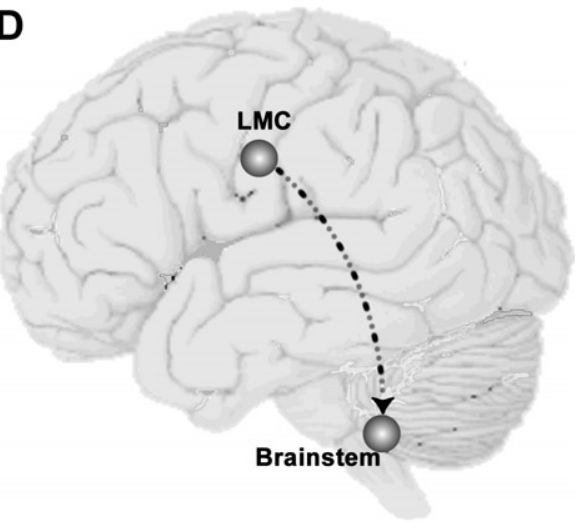

$\mathbf{F}$

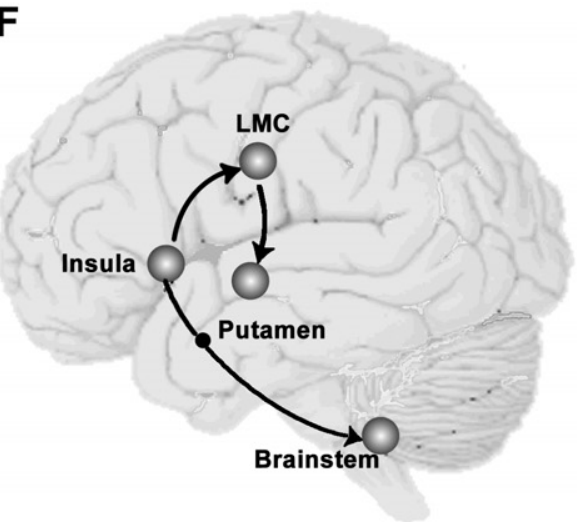

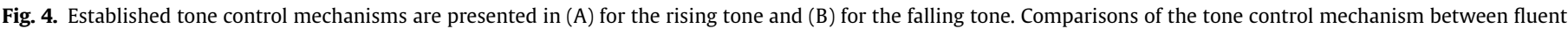

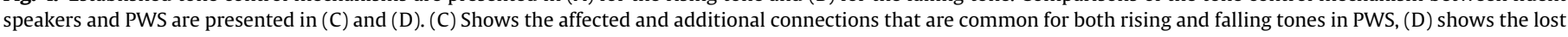

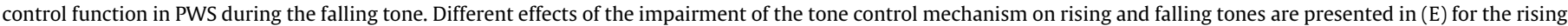
tone and in (F) for the falling tone. The solid lines indicate significance at $p<0.05$ level, whereas the dash lines indicate non-significance.

involving the additional connection in PWS, other possibilities might apply, such as the connections are a reflection of a speechmotor control problem. However, all possible explanations have to be consistent with the finding that although the neural control of both the rising tone and the falling tone showed anomalies, the falling tone appeared to involves more differences between fluent controls and PWS than the rising tone.

\subsection{Laryngeal control of tone in general and in pathological conditions}

Howell et al. (2012) suggested that in fluent speakers during the rising tone, the LMC and the brainstem increased the contraction of the cricothyroid muscle. In the falling tone, the same connection was switched off so that the cricothyroid was relaxed. That was, the switch-on of the connection between the LMC and the brainstem was passively controlled by a negative connection between the left insula and the LMC, whereas the switch-off was actively controlled by a positive connection between the insula and the LMC. It should be noted that the actual mechanism is more complex than that described here. A simplified diagram of these aspects of control is given in Fig. $4 \mathrm{~A}$ and $\mathrm{B}$ for the rising tone and the falling tone respectively.

In PWS, the control connection between the left insula and the LMC differed from that in fluent controls, which would have an impact on both the rising tone and the falling tone. The regions that showed difference were consistent with previous evidence that has shown that the functional and structural connections between the left IFC/insula and the motor cortex are altered in PWS. This 
difference is a reflection of the underlying neural anomalies because an additional connection between the LMC and the putamen was found (see Fig. 4C).

The impairment to the active cessation mechanism would result in loss of control by the connection between the LMC and the brainstem (see Fig. 4D). The control that is lost from the connection between the LMC and the brainstem has different effects on rising and falling tones. That was, although both rising and falling tones required control of the connection between the insula and the LMC, the falling tone needed active cessation-control as established earlier (Howell et al., 2012). The connection between the LMC and the brainstem can function normally in the rising tone (see Fig. 4E). However, during the falling tone, because of the loss of the connection between the LMC and the brainstem, falling-tone control occurs abnormally. This is probably the reason that an additional connection between the insula and the brainstem appeared (see Fig. 4F). This additional direct connection from the insula to the brainstem provides at least part of the active cessation control that was lost for the falling tone, and thus suggests that the falling-tone control should be more affected in PWS than is the rising tone.

\subsection{Limitations}

Several issues need to be noted. First, the present study cannot determine whether the neural control difference between fluent controls and PWS during rising-tone and falling-tone control was the cause or the effect of long-term stuttering. An additional longitudinal study about tone control in Mandarin PWS would be necessary to resolve this issue. Second, although there was a small gender imbalance between the PWS and fluent participants, the PWS sample used here reflected that there are more male than female PWS. Arguably, this makes the PWS sample representative of their corresponding general population that can be compared with the roughly equal gender makeup of the control sample. A third limitation of the current study is that the participants heard continuous scanner noise, which would have masked their productions which, in turn would cause a Lombard effect. Although the participants wore headphones that effectively reduced the background noise during the experiment, influences of speaking environment should be examined in future work. Finally, it would have been helpful if the verbal response had been collected whilst scanning. This would have allowed verification of whether irregularities in tone production occurred in PWS during the imaging experiment and would have allowed examination of whether tone production deficits were manifest behaviorally in stuttering (e.g. were falling tones more affected than rising tones). To this end, recordings will be made in future studies.

\section{Conclusion}

The present study examined speech-motor control of PWS that are associated with rising-tone and falling-tone control mechanisms. The results confirmed the hypothesis that these tone control mechanisms differed between fluent controls and PWS. Moreover, while the altered connection between the insula and the LMC was associated with both rising-tone and falling-tone control, the additional connection between the insula and the brainstem was only associated with falling-tone control in PWS. It is suggested that while both the rising and falling tones showed anomalies in PWS, the falling tone seemed to be more severely affected. These findings raise questions about whether the neural control difference is one that just affects tone control, or whether it is language universal.

\section{Acknowledgments}

This study was supported by grants from the National Natural Science Foundation of China (NSFC) (31270023) and Fundamental Research Funds for the Central Universities (Dr. Chunming Lu) and the Open Research Fund of the State Key Laboratory of Cognitive Neuroscience and Learning (Dr. Peter Howell).

\section{Appendix A. Supplementary data}

Supplementary data associated with this article can be found, in the online version, at http://dx.doi.org/10.1016/j.bandl.2012. 09.010.

\section{References}

Bergmann, G. (1986). Studies in stuttering as a prosodic disturbance. Journal of Speech, Language, and Hearing Research, 29(3), 290-300.

Brown, S., Ingham, R. J., Ingham, J. C., Laird, A. R., \& Fox, P. T. (2005). Stuttered and fluent speech production: An ALE meta-analysis of functional neuroimaging studies. Human Brain Mapping, 25(1), 105-117.

Brown, S., Ngan, E., \& Liotti, M. (2008). A larynx area in the human motor cortex. Cerebral Cortex, 18(4), 837-845.

Büchel, C., Coull, J. T., \& Friston, K. J. (1999). The predictive value of changes in effective connectivity for human learning. Science, 283(5407), 1538-1541.

Chang, S. E., Erickson, K. I., Ambrose, N. G., Hasegawa-Johnson, M. A., \& Ludlow, C. L. (2008). Brain anatomy differences in childhood stuttering. Neuroimage, 39(3), 1333-1344.

Chang, S. E., Horwitz, B., Ostuni, J., Reynolds, R., \& Ludlow, C. L. (2011). Evidence of left inferior frontal-premotor structural and functional connectivity deficits in adults who stutter. Cerebral Cortex.

Chang, S. E., Kenney, M. K., Loucks, T. M. J., \& Ludlow, C. L. (2009). Brain activation abnormalities during speech and non-speech in stuttering speakers. Neurolmage, 46(1), 201-212.

Cox, R. W. (1996). AFNI: Software for analysis and visualization of functional magnetic resonance neuroimages. Computers and Biomedical Research, 29(3), $162-173$.

Cykowski, M. D., Fox, P. T., Ingham, R. J., Ingham, J. C., \& Robin, D. A. (2010). A study of the reproducibility and etiology of diffusion anisotropy differences in developmental stuttering: A potential role for impaired myelination. Neurolmage, 52(4), 1495-1504.

Fiebach, C. J., Friederici, A. D., Smith, E. E., \& Swinney, D. (2007). Lateral inferotemporal cortex maintains conceptual-semantic representations in verbal working memory. Journal of Cognitive Neuroscience, 19(12), 2035-2049.

Forman, S. D., Cohen, J. D., Fitzgerald, M., Eddy, W. F., Mintun, M. A., \& Noll, D. C. (1995). Improved assessment of significant activation in functional magnetic resonance imaging (fMRI): use of a cluster-size threshold. Magnetic Resonance in Medicine, 33(5), 636-647.

Genovese, C. R., Lazar, N. A., \& Nichols, T. (2002). Thresholding of statistical maps in functional neuroimaging using the false discovery rate. Neurolmage, 15(4), 870-878.

Giraud, A. L., Neumann, K., Bachoud-Levi, A. C., von Gudenberg, A. W., Euler, H. A., Lanfermann, H., et al. (2008). Severity of dysfluency correlates with basal ganglia activity in persistent developmental stuttering. Brain and Language, 104(2), 190-199.

Harvey, N., \& Howell, P. (1980). Isotonic vocalis contraction as a means of producing rapid decreases in Fo. Journal of Speech and Hearing Research, 23(3), 576-592.

Howell, P., Jiang, J., Peng, D., \& Lu, C. (2012). Neural control of fundamental frequency rise and fall in Mandarin tones. Brain and Language, 121(1), 35-46.

Howell, P., \& Vause, L. (1986). Acoustic analysis and perception of vowels in stuttered speech. Journal of the Acoustical Society of America, 79(5), 1571-1579.

Howell, P., \& Williams, M. (1988). The contribution of the excitatory source to the perception of neutral vowels in stuttered speech. Journal of the Acoustical Society of America, 84(1), 80-89.

Howell, P., \& Williams, M. (1992). Acoustic analysis and perception of vowels in children's and teenagers' stuttered speech. Journal of the Acoustical Society of America, 91(3), 1697-1706.

Howell, P., \& Young, K. (1990). Analysis of periodic and aperiodic components during fluent and dysfluent phases of child and adult stutterers' speech. Phonetica, 47(3-4), 238-243.

Hua, Z., \& Dodd, B. (2000). The phonological acquisition of Putonghua (Modern Standard Chinese). Journal of Child Language, 27(1), 3-42.

Jöreskog, K. G., \& Sorbom, D. (1996). LISREL 8. User's Reference Guide. Chicago: Scientific Software International.

Loucks, T., Chon, H., \& Han, W. (2012). Audiovocal integration in adults who stutter. International Journal of Language and Communication Disorders, 47(4), 451-456.

Loucks, T. M., \& De Nil, L. F. (2006). Oral kinesthetic deficit in adults who stutter: A target-accuracy study. Journal of Motor Behavior, 38(3), 238-246.

Loucks, T. M., De Nil, L. F., \& Sasisekaran, J. (2007). Jaw-phonatory coordination in chronic developmental stuttering. Journal of Communication Disorders, 40(3), 257-272. 
Lu, C., Chen, C., Ning, N., Ding, G., Guo, T., Peng, D., et al. (2010a). The neural substrates for atypical planning and execution of word production in stuttering. Experimental Neurology, 221(1), 146-156.

Lu, C., Ning, N., Peng, D., Ding, G., Li, K., Yang, Y., et al. (2009). The role of large-scale neural interactions for developmental stuttering. Neuroscience, 161(4), 1008-1026.

Lu, C., Peng, D., Chen, C., Ning, N., Ding, G., Li, K., et al. (2010b). Altered effective connectivity and anomalous anatomy in the basal ganglia-thalamocortical circuit of stuttering speakers. Cortex, 46(1), 49-67.

Ludlow, C. L. (2005). Central nervous system control of the laryngeal muscles in humans. Respiratory Physiology \& Neurobiology, 147(2-3), 205-222.

Max, L., Caruso, A. J., \& Gracco, V. L. (2003). Kinematic analyses of speech, orofacial nonspeech, and finger movements in stuttering and nonstuttering adults. Journal of Speech, Language and Hearing Research, 46(1), 215-232.

Max, L., \& Gracco, V. L. (2005). Coordination of oral and laryngeal movements in the perceptually fluent speech of adults who stutter. Journal of Speech, Language, and Hearing Research, 48(3), 524-542.

McIntosh, A. R., \& Gonzalez-Lima, F. (1994a). Network interactions among limbic cortices, basal forebrain, and cerebellum differentiate a tone conditioned as a Pavlovian excitor or inhibitor: fluorodeoxyglucose mapping and covariance structural modeling. Journal of Neurophysiology, 72(4), 1717-1733.

McIntosh, A. R., \& Gonzalez-Lima, F. (1994b). Structural equation modeling and it application to network analysis in functional brain imaging. Human Brain Mapping, 2, 2-22.

McIntosh, A. R., Grady, C. L., Ungerleider, L. G., Haxby, J. V., Rapoport, S. I., \& Horwitz, B. (1994). Network analysis of cortical visual pathways mapped with PET. Journal of Neuroscience, 14(2), 655-666.

Namasivayam, A. K., \& Van Lieshout, P. (2008). Investigating speech motor practice and learning in people who stutter. Journal of Fluency Disorders, 33(1), 32-51.

Oldfield, R. C. (1971). The assessment and analysis of handedness: The Edinburgh inventory. Neuropsychologia, 9(1), 97-113.

Peschke, C., Ziegler, W., Kappes, J., \& Baumgaertner, A. (2009). Auditory-motor integration during fast repetition: The neuronal correlates of shadowing. NeuroImage, 47(1), 392-402.

Purves, D., Augustine, G. J., Fitzpatrick, D., Hall, W. C., LaMantia, A. S., \& White, L. E. (2011). Neuroscience (5th ed.). USA: Sinauer Associates, Inc.

Riley, G. D. (1994). Stuttering severity instrument for children and adults (3rd ed.). Austin, TX: Pro-Ed.

Salmelin, R., Schnitzler, A., Schmitz, F., \& Freund, H. J. (2000). Single word reading in developmental stutterers and fluent speakers. Brain, 123(Pt 6), 1184-1202.

Shi, Y., \& Li, S. L. (2011). Mechanisms and performance of voice disorders in aphasia (review). Chinese Journal of Rehabilitation Theory and Practice, 17(2), 148-150.

Simmonds, A. J., Wise, R. J., Dhanjal, N. S., \& Leech, R. (2011). A comparison of sensory-motor activity during speech in first and second languages. Journal of Neurophysiology, 106(1), 470-478.

Simonyan, K., \& Horwitz, B. (2011). Laryngeal motor cortex and control of speech in humans. The Neuroscientist, 17(2), 197-208.

Simonyan, K., Ostuni, J., Ludlow, C. L., \& Horwitz, B. (2009). Functional but not structural networks of the human laryngeal motor cortex show left hemispheric lateralization during syllable but not breathing production. The Journal of Neuroscience, 29(47), 14912-14923.
Smith, A. (1989). Neural drive to muscles in stuttering. Journal of Speech, Language, and Hearing Research, 32(2), 252-264.

Smith, A., Denny, M., Shaffer, L. A., Kelly, E. M., \& Hirano, M. (1996). Activity of intrinsic laryngeal muscles in fluent and disfluent speech. Journal of Speech, Language, and Hearing Research, 39(2), 329-348.

Smith, A., \& Kleinow, J. (2000). Kinematic correlates of speaking rate changes in stuttering and normally fluent adults. Journal of Speech, Language, and Hearing Research, 43(2), 521-536.

Smith, A., Luschei, E., Denny, M., Wood, J., Hirano, M., \& Badylak, S. (1993). Spectral analyses of activity of laryngeal and orofacial muscles in stutterers. Journal of Neurology, Neurosurgery and Psychiatry, 56(12), 1303-1311.

Smith, A., Sadagopan, N., Walsh, B., \& Weber-Fox, C. (2010). Increasing phonological complexity reveals heightened instability in inter-articulatory coordination in adults who stutter. Journal of Fluency Disorders, 35(1), 1-18.

Smits-Bandstra, S., \& De Nil, L. F. (2007). Sequence skill learning in persons who stutter: implications for cortico-striato-thalamo-cortical dysfunction. Journal of Fluency Disorders, 32(4), 251-278.

Sommer, M., Koch, M. A., Paulus, W., Weiller, C., \& Buchel, C. (2002). Disconnection of speech-relevant brain areas in persistent developmental stuttering. Lancet, 360(9330), 380-383.

Van Lieshout, P. H., Hulstijn, W., \& Peters, H. F. (1996). Speech production in people who stutter: Testing the motor plan assembly hypothesis. Journal of Speech, Language, and Hearing Research, 39(1), 76-92.

Van Lieshout, P. H., Peters, H. F., Starkweather, C. W., \& Hulstijn, W. (1993). Physiological differences between stutterers and nonstutterers in perceptually fluent speech: EMG amplitude and duration. Journal of Speech, Language, and Hearing Research, 36(1), 55-63.

Viswanath, N. S., \& Neel, A. T. (1995). Part-word repetitions by persons who stutter: Fragment types and their articulatory processes. Journal of Speech, Language, and Hearing Research, 38(4), 740-750.

Watkins, K. E., Smith, S. M., Davis, S., \& Howell, P. (2008). Structural and functional abnormalities of the motor system in developmental stuttering. Brain, 131(Pt 1), 50-59.

Wu, J. C., Maguire, G., Riley, G., Fallon, J., LaCasse, L., Chin, S., et al. (1995). A positron emission tomography [18F]deoxyglucose study of developmental stuttering. Neuroreport, 6(3), 501-505.

Wu, J. C., Maguire, G., Riley, G., Lee, A., Keator, D., Tang, C., et al. (1997). Increased dopamine activity associated with stuttering. Neuroreport, 8(3), 767-770.

Xiong, J., Gao, J. H., Lancaster, J. L., \& Fox, P. T. (1995). Clustered pixels analysis for functional MRI activation studies of the human brain. Human Brain Mapping, 3, 287-301.

Xu, Y., \& Liu, F. (2012). Intrinsic coherence of prosodic and segmental aspects of speech. In O. Niebuhr (Ed.), Prosodies - Context, function, communication (pp. 1-26). Berlin/New York: de Gruyter.

Xu, Y., \& Wang, Q. E. (2001). Pitch targets and their realization: Evidence from Mandarin Chinese. Speech Communication, 33(4), 319-337.

Yip, M. (2002). Tone Cambridge textbooks in linguistics. Cambridge: Cambridge University Press.

Zhang, Q., \& Yang, Y. (2003). The determiners of picture-naming latency. Acta Psychologica Sinica, 35(4), 447-454. 